\title{
Non-linear Distortion Noise Cancellation for Satellite Forward Links
}

\author{
Svilen Dimitrov \\ German Aerospace Center (DLR), Satellite Networks Department, 82234 Wessling, Germany, e-mail: svilen.dimitrov@dlr.de
}

\begin{abstract}
In this paper, an iterative receiver that performs non-linear distortion noise cancellation is proposed for application at the user terminal in the DVB-S2X satellite forward link. The performance is assessed for a single-carrier time-division multiplexing (TDM) waveform with low carrier roll-off factors, e.g., down to $5 \%$, and high baud rates, e.g., up to $34 \mathrm{MBaud}$ in a $36-\mathrm{MHz}$ transponder, in accordance with a very-small-aperture terminal (VSAT) scenario. The satellite forward-link channel is comprehensively modelled, including the input multiplexing (IMUX) and output multiplexing (OMUX) filter responses at the satellite transponder, the non-linear travelling wave tube amplifier (TWTA) characteristics, and the phase noise at the user terminal. Modulation orders up to 256-level amplitude and phase-shift keying (APSK) are evaluated with low-density paritycheck (LDPC) forward error correction (FEC). The improved receiver is compared to state-of-the-art compensation techniques, such as pre-distortion at transmitter and linear equalization at receiver. In the higher-baud-rate scenario, the improved receiver demonstrates a 5.2-dB increase of the energy efficiency at 32 APSK or up to $40 \%$ increase of the spectral efficiency. In the lower-baud-rate scenario, up to 1.8-dB increase of the energy efficiency at 256-APSK is presented.

Index Terms-Satellite communications, non-linear distortion,
\end{abstract} iterative receiver, equalization, spectral efficiency.

\section{INTRODUCTION}

The recently published DVB-S2X standard [1] introduces a number of extensions for the air interface of the satellite forward link: 1) wide-band operation with transponder bandwidths up to $250 \mathrm{MHz}$ for application with Ka-band transponders, 2) higher-order amplitude and phase-shift keying (APSK) modulation up to 256-APSK and additional low-density paritycheck (LDPC) codes for more efficient resource utilization with finer signal-to-noise ratio (SNR) threshold granularity, and 3 ) lower roll-off factors down to $5 \%$ with higher symbol rates in order to increase the spectral efficiency. A number of scenarios in state-of-the-art satellite communication systems resort to single-carrier utilization of the satellite transponder bandwidth [2], e.g., in very-small-aperture terminal (VSAT) or direct-to-home (DTH) applications. However, due to the imperfect magnitude and group-delay responses of the inputmultiplexing (IMUX) and output-multiplexing (OMUX) filters on board the satellite, linear distortion is introduced in the form of inter-symbol interference (ISI). In addition, due to the power limitation of the satellite, the travelling wave tube amplifier (TWTA) is operated close to saturation. Because of the nonlinear transfer characteristic of the TWTA, non-linear distortion, in the form of constellation warping and clustering at the receiver demodulator, is introduced when waveforms with high peak-to-average-power ratio (PAPR) are used for transmission, e.g., pules-shaped time-division multiplexing (TDM) employ- ing constellations up to 256-APSK with multiple amplitude rings. Without adequate channel compensation, these adverse effects can reduce the energy efficiency and spectral efficiency gains expected with the application of tighter roll-off factors, higher symbol rates, and higher modulation orders.

A linear equalizer has been recently introduced in the DVB$\mathrm{S} 2 \mathrm{X}$ receiver [2] to counter the ISI. The non-linear distortion can be handled by means of output back-off (OBO) adjustment [3]. This approach is known to penalize the energy efficiency of the system when high OBO is applied. In addition, a number of pre-distortion techniques at the transmitter can be employed, such as data pre-distortion [3], [4], [5], [6] or signal pre-distortion [4], [7], and static data pre-distortion [3], [4] is the state-of-the-art solution in DVB-S2X. Decision-directed advanced equalization techniques [8] applied at the receiver are known to provide very good performance in non-linear channels with memory [9], such as a turbo equalizer based on a Volterra series representation of the channel. Here, a major reduction of the computational complexity can be achieved by using first-order decomposition of a set of received symbols at the input of the demodulator, e.g., a buffered physical layer (PL) frame, in a set of first-order symbol-based constellation scaling factors representing the constellation warping effects plus a non-linear noise component containing the higher nonlinear orders [10]. This decomposition can be used to reconstruct and cancel the interfering component, using channel models based on the measured responses of the deterministic distortion sources along the chain, such as IMUX/OMUX responses and TWTA transfer characteristics.

In this paper, symbol-based equalization with non-linear noise cancellation is proposed for the satellite forward link as an addition to the standard linear equalizer at the receiver. Comprehensive modelling of the Ka-band satellite forward link channel is performed, including the IMUX and OMUX filter magnitude and group-delay responses, the TWTA amplitude and phase transfer characteristics, the aggregate phase noise power spectral density mask of the up- and downconversion stages, and the additive white Gaussian noise (AWGN) at the low-noise amplifier (LNA) of the receiver. The performance of the novel receiver is evaluated for TDM with a low roll-off factor of $5 \%$ and a higher baud rate of 34 MBaud in a $36-\mathrm{MHz}$ transponder, and it is compared to the standard $20 \%$ roll-off with a lower baud rate of 27.5 MBaud. Modulation orders up to 256-APSK are evaluated with LDPC forward error correction (FEC). The improved receiver provides significant gains in energy efficiency and 


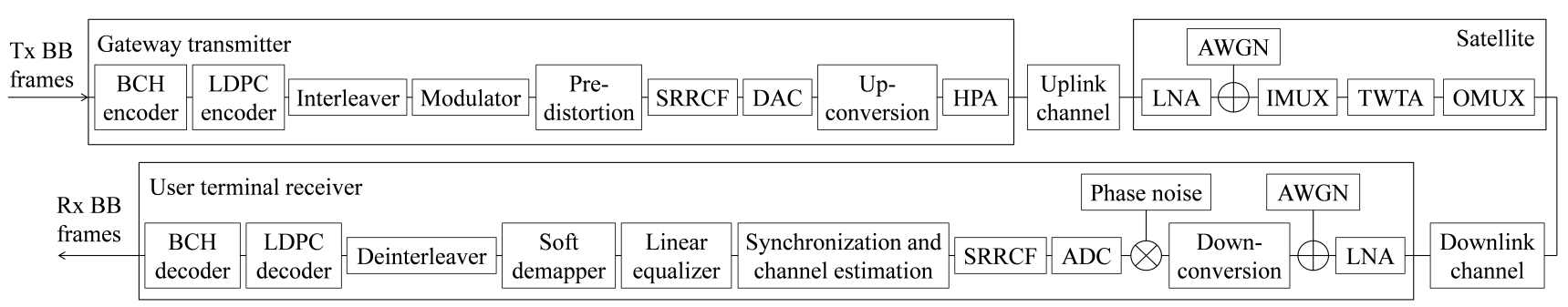

Fig. 1: Block diagram of the satellite transmission chain in the forward link.

spectral efficiency as compared to standard static data predistortion and linear equalization [1]. Modulation orders of 64-APSK, 128-APSK, and 256-APSK are enabled to meet the packet-error rate (PER) target in the scenario with $5 \%$ roll-off and higher baud rate, resulting in $5.2-\mathrm{dB}$ increase of energy efficiency relative to 32 -APSK or up to $40 \%$ increase of spectral efficiency. In the scenario with $20 \%$ roll-off and a lower baud rate, up to $1.8-\mathrm{dB}$ reduction of the energy requirement is presented. The gains increase for higher order modulations due to their higher sensitivity to non-linear distortion.

The rest of the paper is organized as follows. Section II describes the satellite system model, including the transmission chain, the channel modelling, and the state-of-the-art channel compensation techniques. Section III describes the operation of the symbol-based equalizer with non-linear noise cancellation. Section IV presents the performance evaluation results. Finally, Section V concludes the paper.

\section{Satellite System Model}

In the satellite forward link, a gateway station relays messages to user terminals over a satellite. In this section, the satellite transmission chain, the channel model, and the stateof-the-art channel compensation techniques are presented.

\section{A. Satellite Transmission Chain}

The block diagram of the satellite transmission chain is presented in Fig. 1. At the gateway transmitter, a stream of data packets with 1504 bits per packet are encoded by means of a Bose-Chaudhuri-Hocquenghem (BCH) code and an LDPC code, followed by a bit interleaver. The resulting FEC frame with length of up to 64800 bits is mapped to symbols, using constellations up to 256-APSK at the modulator. Static data pre-distortion [3], [4] is used in DVB-S2X to reduce the non-linear distortion. The symbols are grouped in bundled physical-layer (PL) frames with up to 67920 symbols per PL frame, which are in term grouped in superframes with 612540 symbols per superframe. The symbols are pulse shaped by a square root raised cosine filter (SRRCF), and after digital-toanalog conversion (DAC), the baseband signal is up-converted to the carrier frequency, e.g., in the Ka band. Finally, the signal is amplified by means of a high-power amplifier (HPA), and is transmitted over the feeder uplink.

At the transponder onboard the satellite, the signal is amplified by an LNA, and AWGN is introduced. Next, the signal is passed through an IMUX filter, a high-power TWTA, and an OMUX filter. Here, the imperfect frequency responses of the IMUX and OMUX filters introduce linear distortion in the form of ISI, while the TWTA introduces non-linear distortion. The signal is then transmitted over the user downlink.

At the user terminal receiver, the signal is amplified by an LNA, and AWGN is introduced. During down-conversion to baseband, the signal is distorted by phase noise. Next, an analog-to-digital converter (ADC) and a matched SRRCF are applied. After timing and frame synchronization, and channel estimation, the symbols are passed through a linear equalizer to counter the linear distortion. A fractionally-spaced adaptive linear equalizer with 42 complex-valued taps is applied, working at twice the symbol rate. The symbol vector at the output of the linear equalizer can be expressed as $\mathbf{x}^{(0)}=\operatorname{Eq}\{\mathbf{y}\}=\mathbf{e} * \mathbf{y}$, where $\operatorname{Eq}\{\cdot\}$ is the equalizer operator, $*$ is the linear convolution operator, $\mathbf{y}$ is the symbol vector at the input of the linear equalizer, and $\mathbf{e}$ is the vector with the equalizer taps. The taps of the adaptive equalizer are updated in a data-aided manner over pilot symbols, using the following recursive algorithm [8]:

$$
\mathbf{e}_{k+1}=\mathbf{e}_{k}-\mu\left(\mathbf{e}_{k} \mathbf{y}_{k}^{\mathrm{T}}-x_{k}\right) \mathbf{y}_{k}^{*}
$$

where $\mathbf{e}_{k+1}$ is the updated equalizer tap vector after the $k$ th pilot $x_{k}, \mathbf{e}_{k}=\left[e_{0}, e_{1}, \ldots, e_{N_{\mathrm{EQ}}-1}\right]$ contains $N_{\mathrm{EQ}}$ taps, initialized as the zero vector $\mathbf{e}_{0}=[0, \ldots, 0]$. The window vector containing $N_{\mathrm{EQ}}$ samples of the received symbol vector $\mathbf{y}$ is denoted as $\mathbf{y}_{k}=\left[y_{k}, y_{k-1}, \ldots, y_{k-N_{\mathrm{EQ}}}\right]$, and is initialized as $\mathbf{y}_{0}=\left[y_{0}, 0, \ldots, 0\right]$. Here, $(\cdot)^{\mathrm{T}}$ is the transpose operator, and $(\cdot)^{*}$ denotes complex conjugation. With values between 0 and 1 , the step size $\mu$ represents a trade-off between convergence speed and stability, e.g., $\mu=0.005$. After the linear equalization, the symbols are downsampled to the Nyquist rate. Finally, the demodulator and the decoder recover the received data packets. Here, a soft demapper generates log-likelihood ratios (LLRs), which after deinterleaving are provided as input to the LDPC decoder, followed by a BCH decoder.

A number of simplifications of the satellite transmission chain in the forward link can be considered, preserving all key aspects affecting the physical layer performance. The free-space propagation can be characterized as a slow-fading process due to rainy weather. It is handled by means of adaptive coding and modulation (ACM), as well as a fading margin in the link budget. The feeder link is generally not power limited, and therefore the AWGN after the onboard LNA is negligible. Since the HPA at the gateway is operated in the linear region, the nonlinearity in the satellite channel 
is introduced primarily by the TWTA. The transmitter and receiver are assumed to be fine synchronized, i.e., genie-aided synchronization is performed based on correlation with the transmitted signal, ideally assumed to be known. As a result, the received PL frame at the input of the demodulator at the user terminal receiver can be expressed as:

$$
\begin{aligned}
\mathbf{x}^{(0)}= & \operatorname{Eq}\left\{\mathbf { h } _ { \mathrm { SRRCF } } * \left[\mathbf { h } _ { \mathrm { OMUX } } * F \left(\mathbf{h}_{\mathrm{IMUX}} *\right.\right.\right. \\
& \left.\left.\left.* \mathbf{h}_{\mathrm{SRRCF}} * \operatorname{Pre}\{\mathbf{x}\}\right)+\mathbf{w}\right] \circ \exp (-j \boldsymbol{\psi})\right\},
\end{aligned}
$$

where $\mathbf{x}$ is the transmitted symbol vector, Pre $\{\cdot\}$ is the applied pre-distortion operator, $\mathbf{h}_{\mathrm{SRRCF}}$ is the impulse response vector of the SRRCF filter, and $\mathbf{h}_{\text {IMUX }}$ is the impulse response of the IMUX filter. The non-linear transfer function of the TWTA is denoted as $F(\cdot), \mathbf{h}_{\text {OMUX }}$ is the impulse response of the OMUX filter, $w$ is the AWGN vector, $\boldsymbol{\psi}$ is the phase deviation vector, and $\circ$ denotes element-wise multiplication.

\section{B. Satellite Channel Modelling}

In the satellite forward link, the main distortion sources are the imperfect characteristics of the IMUX and OMUX filters and the TWTA nonlinearity. The signal is also distorted by AWGN at the receiver LNA, and phase noise is introduced due to oscillator instabilities during up- and down-conversions.

1) IMUX and OMUX Filters: A suitable representation of the IMUX and OMUX responses is obtained by means of a stable Chebyshev Type II infinite impulse response (IIR) filter. The synthesized magnitude response and group-delay response of the IMUX and OMUX filters are presented in Fig. 2, using the cheby2 function of the MathWorks MATLAB signal processing toolbox. A 7th-order Chebyshev Type II filter with $-34-\mathrm{dB}$ ripple and $23-\mathrm{MHz}$ edge frequency is used for the IMUX filter, while a 5th-order Chebyshev Type II filter with $-38-\mathrm{dB}$ ripple and $28.6-\mathrm{MHz}$ edge frequency is considered for the OMUX filter. They show to be a good match when compared to the measured responses from [2]. The resulting digital impulse responses, $\mathbf{h}_{\text {IMUX }}$ and $\mathbf{h}_{\text {OMUX }}$, are convolved with a digital representation of the signal. The IMUX/OMUX filters introduce linear distortion in the form of memory effects due to frequency selectivity at high baud rates, which corrupts the matched filtering at the receiver, and amplifies the AWGN.

2) TWTA: In this study, a practical model of a linearized Ka-band TWTA is considered. The input amplitude/output amplitude (AM/AM) and input amplitude/output phase (AM/PM) characteristics are presented in Fig. 3, and are denoted as $A(\cdot)$ and $\phi(\cdot)$, respectively. They only depend on the signal amplitude. As a result, the output signal after the TWTA, $F(s)$, can be expressed as $F(s)=A(|s|) \exp [j \phi(|s|)+j \arg (s)]$, where $s$ is the input signal. In this study, $F(s)$ is evaluated using linear interpolation or extrapolation on the measured $\mathrm{AM} / \mathrm{AM}$ and AM/PM characteristics.

The non-linear distortion introduces constellation warping and symbol clustering at the input of the demodulator [3]. The interfering component is dependent on the signal waveform, and therefore contains useful information. The received signal can be decomposed in an attenuated and rotated replica of the transmitted signal plus non-linear distortion noise. Each
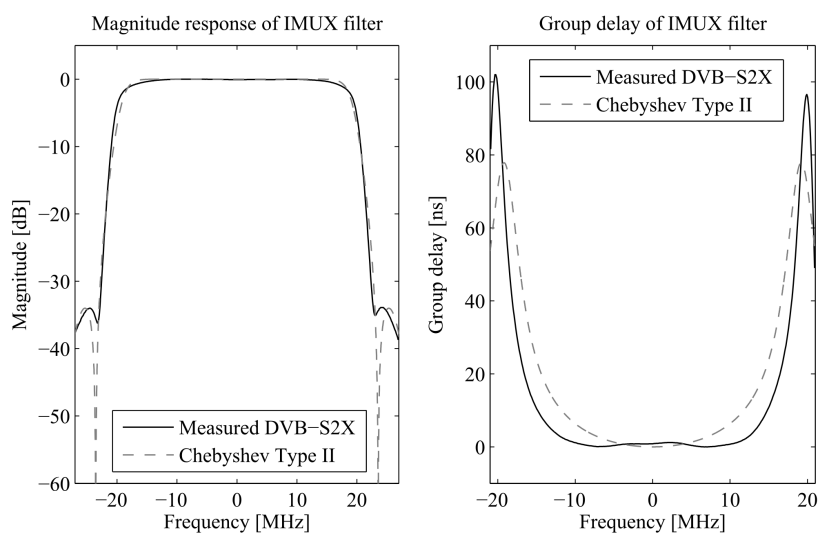

(a) Magnitude and group delay responses of IMUX filter.
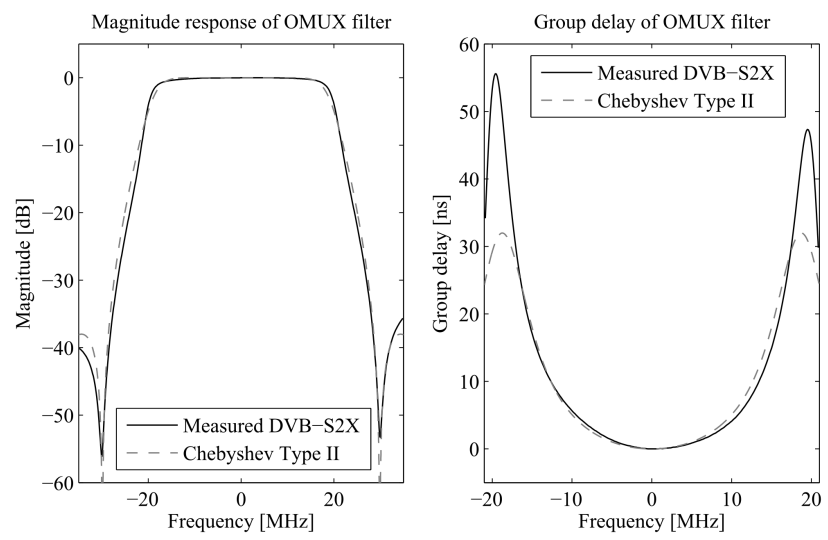

(b) Magnitude and group delay responses of OMUX filter.

Fig. 2: Filter characteristics of a $36-\mathrm{MHz}$ satellite transponder in the forward link.

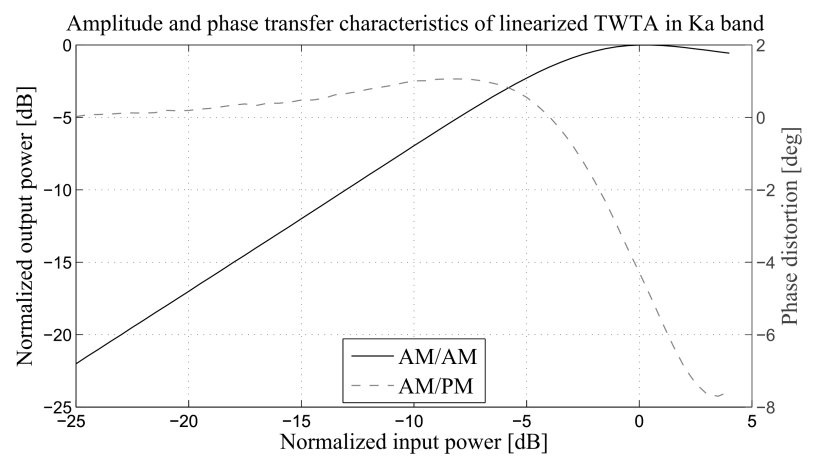

Fig. 3: AM/AM and AM/PM transfer characteristics of a Kaband linearized TWTA.

received centroid can be individually warped, i.e., it is scaled by a complex-valued factor relative to the transmitted constellation point. There is no general assumption on the distribution of the non-linear distortion noise.

3) AWGN and Phase Noise: At the user terminal receiver, the RF bandpass signal is first amplified by an LNA, whereby AWGN with double-sided power spectral density $N_{0} / 2$ is introduced. The equivalent baseband signal collects AWGN with one-sided power spectral density $N_{0}$ [8]. After the downconversion stage, due to local oscillator instabilities, the signal 


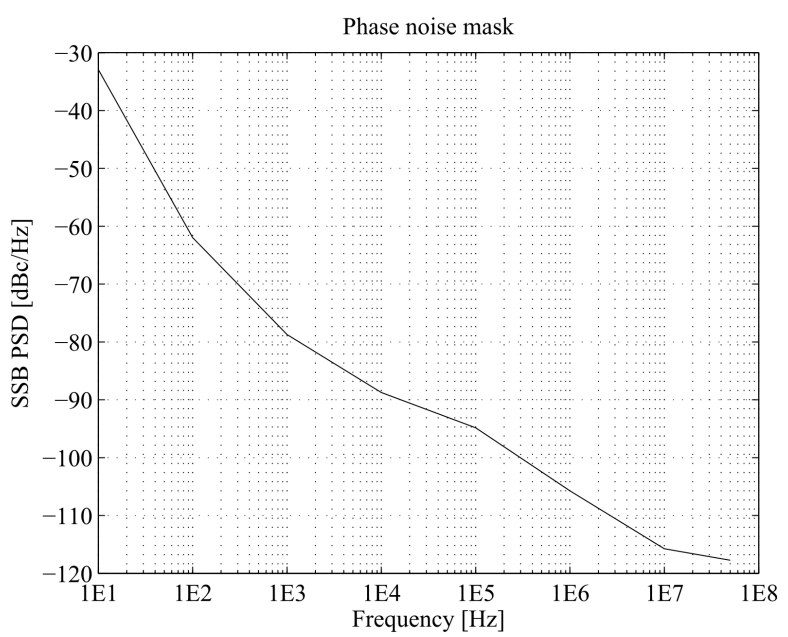

Fig. 4: Phase noise power spectral density mask of the Kaband VSAT scenario in the satellite forward link.

is distorted by phase noise with aggregate single-sided power spectral density mask presented in Fig. 4 according to the Ka-band VSAT scenario [2]. A procedure for generating the phase deviation process, modelled as a Wiener process, i.e., a Gaussian random walk, is outlined in Annex G of [2]. Under the assumption of small signals, the double-sided power spectral density of the Wiener process corresponds to the single-sided power spectral density mask of the phase noise. The generated phase deviation samples modulate a complex phasor, which is then multiplied with the signal.

\section{State-of-the-Art Channel Compensation Techniques}

The non-linear compensation solutions include a variety of pre-distortion techniques at the transmitter. Signal predistortion [4] is commonly used for amplifier linearization by means of analog electronics that implement the inverse of the amplifier characteristic. In addition, signal pre-distortion can also be applied digitally, i.e., on the samples of a pulse shaped signal immediately before the DAC in the transmitter chain. Signal pre-distortion is known to introduce unwanted out-of-band radiation to be suppressed by a transmit filter. A recently proposed solution against spectral regrowth is bandlimited signal pre-distortion [7] which applies digital filters to the out-of-band components and compensates the effect of the non-linear device on the in-band portion of the signal.

Data pre-distortion [3], [4], [5], [6] is a purely digital technique that preserves the signal spectrum. In static data pre-distortion [3], [4], the transmitted constellation is modified such that the received centroids are close to the original transmitted constellation. The pre-distortion factors for each used constellation are stored in a look-up table (LUT). Dynamic data pre-distortion [5], [6] takes into account the current symbol, as well as $(L-1) / 2$ symbols preceding and succeeding each symbol, where $L$ is the memory depth. As a result, the size of the LUT is increased to $M^{L-1}$, where $M$ is the modulation order, for every utilized constellation. Given the already large number of constellations introduced in DVB$\mathrm{S} 2 \mathrm{X}$, the size of the look-up table may require impractically large storage memory. The dynamic pre-distorter functions can also be computed adaptively, using indirect learning [5] or direct learning algorithms [6]. For this purpose, a model for the non-linear channel with memory is required, e.g., a Volterra series or a memory polynomial model [5], [6].

The total distortion at the receiver is the summation of the interfering component due to the non-linear channel with memory, the AWGN, and the phase noise. The pre-distortion techniques aim to minimize the influence only of the interfering component. Decision-directed equalizers implemented in the receiver [8] can further operate jointly with the AWGN and phase noise due to the employed signal detection. The symbol-based equalizer with non-linear noise cancellation studied in this paper is a form of decision-directed equalizer. It employs iterative detection, estimation, and cancellation of the interfering component at the receiver which provides significant energy efficiency gains.

\section{ITERATIVE EQUALIZATION AND CANCELLATION OF NON-LINEAR DISTORTION}

Cancellation of non-linear distortion noise has been shown to be an effective technique to extract useful information from the interfering component and significantly reduce the SNR penalty with respect to the linear channel [10]. In this section, two variations of the non-linear equalizer are proposed for application at the user terminal in the satellite forward link.

\section{A. Background and Motivation}

Decision-directed detection by means of turbo equalization has been proposed for application over non-linear satellite channels in [9]. A channel model based on a Volterra series is assumed, and minimum mean-squared error (MMSE) filters are computed to counter every term in the Volterra series model. A major reduction in complexity can be achieved by means of a simplified model for the received symbols after the non-linear device. Instead of computing MMSE filters for every component in the Volterra series, a simple procedure can be applied to reconstruct and cancel the non-linear noise component. At the heart of the simplification is a first-order decomposition of the received symbols at the input of the demodulator, based on a symbol scaling factor representing the warping effect and a non-linear noise component containing the higher non-linear orders. In addition to non-linear compensation, memory effects in the form of ISI are also taken into account in the reconstruction and cancellation of the interfering component due to the fact that the equalizer operates on a large block of symbols, e.g., a PL frame. The application of the proposed equalizer at the receiver is complementary to any non-linear compensation technique at the transmitter, e.g., pre-distortion, and provides additional gain due to the applied non-linear noise cancellation.

\section{B. Function}

The block diagram of the symbol-based equalizer with nonlinear noise cancellation is presented in Fig. 5. The symbols at the output of the linear equalizer, downsampled to the 


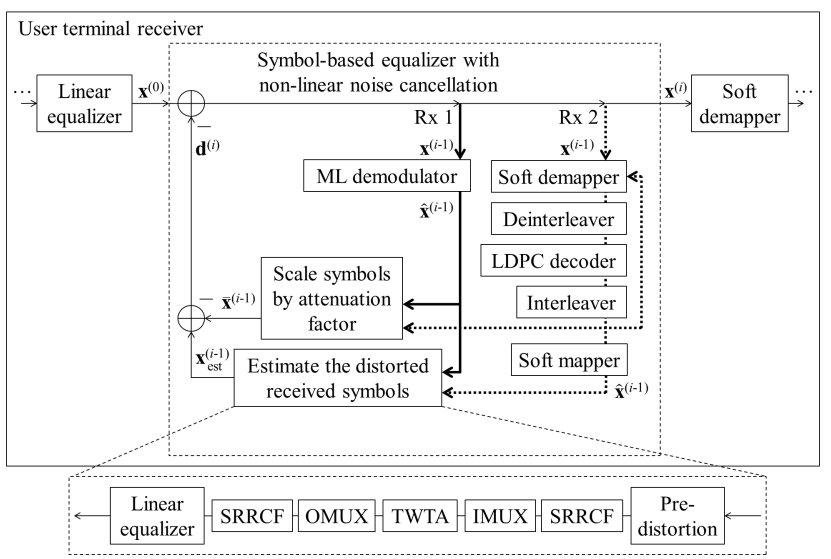

Fig. 5: Block diagram of symbol-based equalizer with nonlinear noise cancellation in the satellite forward link, using an ML demodulator (Rx 1 - bold solid lines) or an LDPC decoder (Rx 2 - bold dotted lines) in the cancellation loop.

Nyquist rate, are provided as input to the iterative nonlinear equalizer. At this point, a set of received symbols, e.g., a received PL frame $\mathbf{x}^{(0)}$, is buffered. As a first step of the processing, detection is performed, whereby two receiver structures are evaluated in this study. The performance of a simple ML demodulator in the detector in the cancellation loop is compared against an LDPC decoder, in order to show the robustness of the proposed equalizer to decision errors.

In the first receiver ( $\mathrm{Rx} 1)$, the symbols are ML demodulated, using the received constellation centroids as a reference. In order to estimate the received constellation centroids, there are dedicated P2 modulated pilot symbols in each DVB-S2X bundled PL frame [1]. The vector of detected symbols used in the $i$ th iteration is denoted as $\widehat{\mathbf{x}}^{(i-1)}=\operatorname{Demod}\left[\mathbf{x}^{(i-1)}\right]$.

In the second receiver ( $\mathrm{Rx} 2)$, the LDPC decoder is included in the cancellation loop in accordance with the setup of the turbo equalizer [9]. The detected symbols are therefore obtained, after soft demapping, deinterleaving, LDPC decoding, interleaving and soft mapping are applied to the symbols $\mathbf{x}^{(i-1)}$. Here, the soft demapper provides extrinsic LLRs to the decoder via deinterleaver, using the received constellation centroids as reference, and employs the interleaved LLRs from the previous decoding iteration as a priori information [9]. The interleaved decoder LLRs are also used in the soft mapper to compute bit probabilities $\operatorname{Pr}\left\{a_{n, q}=b_{m, q}\right\}$, where $a_{n, q}$ are the bits corresponding to a symbol $x_{n}^{(i-1)}, b_{m, q}$ are interleaved source bits corresponding to a constellation point, $c_{m}, q=1, \ldots, \log _{2}(M)$, and $m=1, \ldots, M$. Under the assumption of bit independence, these probabilities are used to compute soft detected symbols as follows:

$$
\widehat{x}_{n}^{(i-1)}=\sum_{m=1}^{M} c_{m} \prod_{q=1}^{\log _{2}(M)} \operatorname{Pr}\left\{a_{n, q}=b_{m, q}\right\} .
$$

The estimate of the interfering component is obtained through subtraction of two terms. To obtain the first term, in Rx 1, the detected symbols are scaled by individual attenuation factors, $K_{c_{m}}$, according to the respective constellation point $c_{m}$, to obtain $\overline{\mathbf{x}}^{(i-1)}$, where $\bar{x}_{c_{m}, n}^{(i-1)}=K_{c_{m}} \widehat{x}_{c_{m}, n}^{(i-1)}$. This is essential to account for the constellation warping effects. The individual scaling factors represent the ratio of a received centroid to the corresponding transmitted constellation point. In $\mathrm{Rx} 2$, soft scaled symbols are calculated as follows:

$$
\bar{x}_{n}^{(i-1)}=\sum_{m=1}^{M} K_{c_{m}} c_{m} \prod_{q=1}^{\log _{2}(M)} \operatorname{Pr}\left\{a_{n, q}=b_{m, q}\right\} .
$$

To obtain the second term, the symbols $\widehat{\mathbf{x}}^{(i-1)}$ are used to obtain an estimate of the received symbols based on models of the distortion functions and the processing blocks along the transmission chain. Here, only models for the deterministic distortion sources are used. These include the IMUX filter, the TWTA, and the OMUX filter. In state-of-the-art non-linear compensation techniques, the Volterra series and memory polynomial models [5], [6] are used to describe the non-linear channel with memory. These models are obtained by means of training data, and facilitate the computation of the channel inverse operation. In general, the proposed equalizer can also apply the Volterra series or memory polynomial models to obtain an estimate of the received symbols. However, since no inverse operation is required here, more accurate models for the linear and non-linear distortion functions can be used, such as the AM/AM and AM/PM characteristics of the TWTA and the magnitude and group-delay responses of the IMUX and OMUX filters, all measured before commission of each satellite. If the joint application of a pre-distorter and the nonlinear equalizer is desired, the setup of the pre-distorter is to be made known to the receiver, e.g., as information in the frame transmission preamble, frame header, or dedicated signalling channel. For completeness, the pre-distortion block is included in the setup of the non-linear equalizer in Fig. 5.

To estimate the received symbols, the symbols $\widehat{\mathbf{x}}^{(i-1)}$ are first passed through the known pre-distorter block. The symbols are then pulse shaped, and the models for the IMUX filter, TWTA (maintaining the operating point), and OMUX filter are applied. After matched filtering, the known linear equalizer block is applied, and the estimate is obtained as:

$$
\begin{aligned}
\mathbf{x}_{\mathrm{est}}^{(i-1)}= & \operatorname{Eq}\left\{\mathbf{h}_{\mathrm{SRRCF}} * \mathbf{h}_{\mathrm{OMUX}} *\right. \\
& \left.* F\left(\mathbf{h}_{\mathrm{IMUX}} * \mathbf{h}_{\mathrm{SRRCF}} * \operatorname{Pre}\left\{\widehat{\mathbf{x}}^{(i-1)}\right\}\right)\right\} .
\end{aligned}
$$

The scaled symbols are subtracted from the estimate of the received symbols to reconstruct an estimate of the non-linear distortion noise as:

$$
\mathbf{d}^{(i)}=\mathbf{x}_{\mathrm{est}}^{(i-1)}-\overline{\mathbf{x}}^{(i-1)} .
$$

This estimate of the interfering component is subtracted from the buffered symbols to obtain a new set of the received symbols as an output of the first iteration of non-linear noise cancellation. In the second iteration, the newly obtained received symbols are detected and used to better estimate the interfering component, which is again subtracted from the 


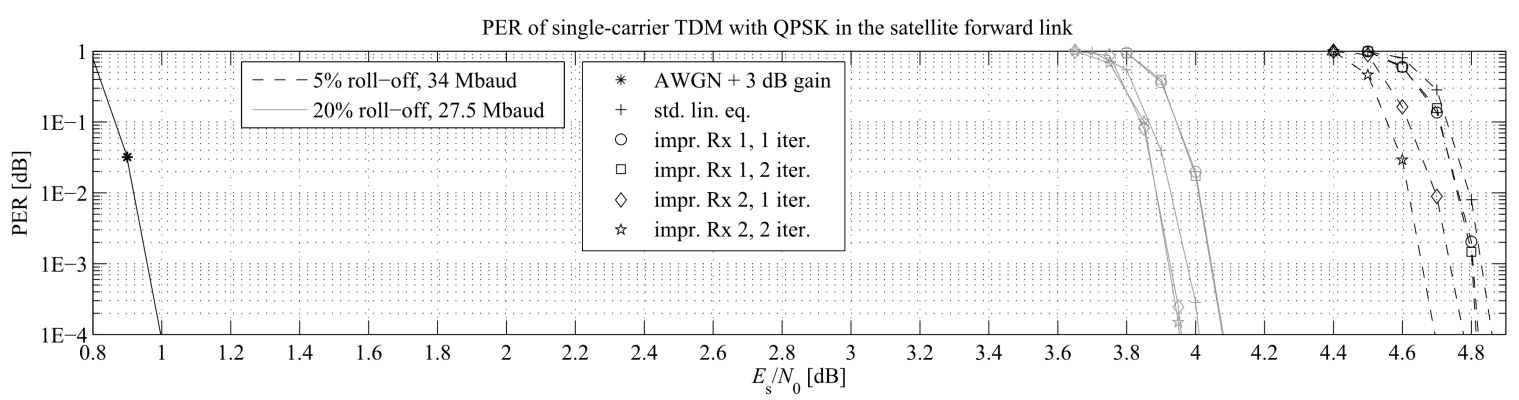

(a) PER of single-carrier TDM with QPSK with 3/4-rate LDPC code and BCH code.

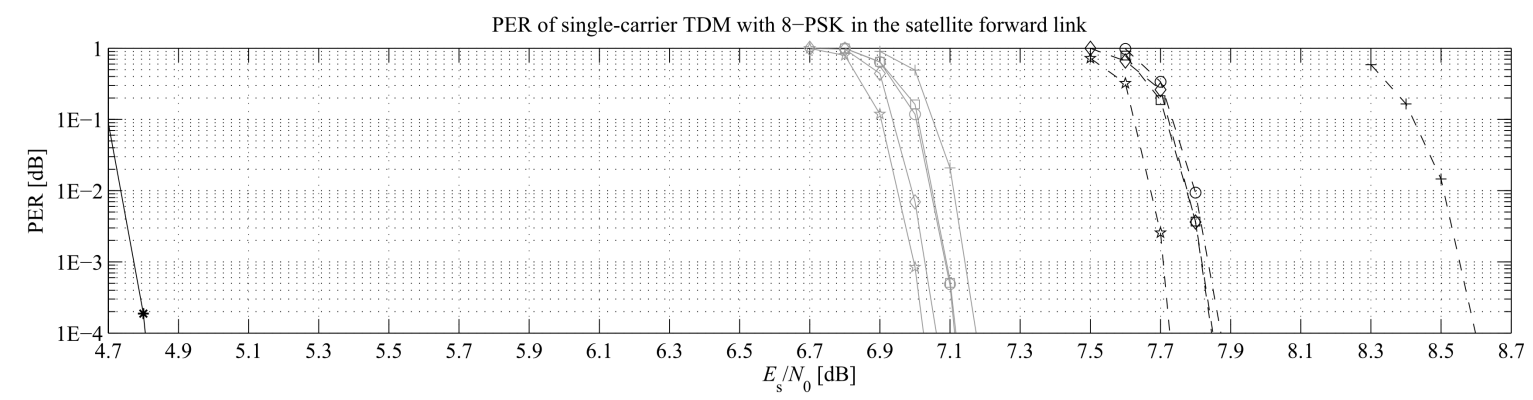

(b) PER of single-carrier TDM with 8-PSK with 3/4-rate LDPC code and BCH code.

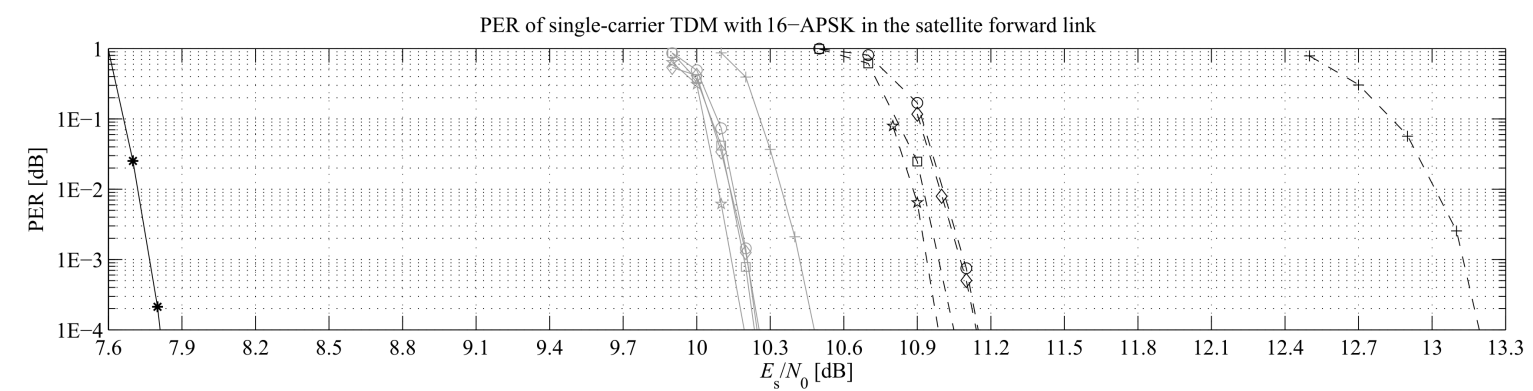

(c) PER of single-carrier TDM with 16-APSK with 3/4-rate LDPC code and BCH code.

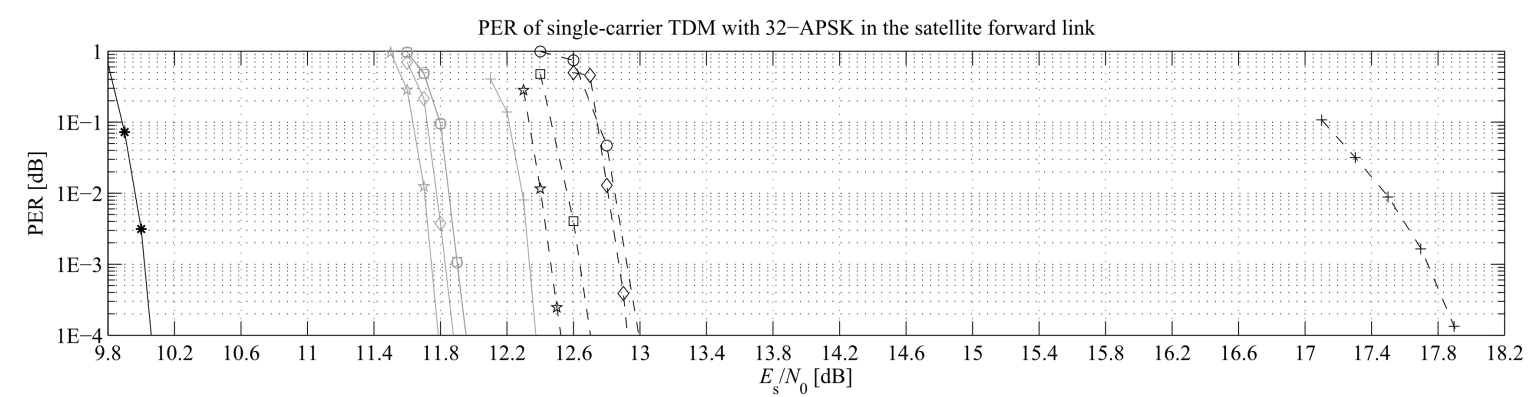

(d) PER of single-carrier TDM with 32-APSK with 3/4-rate LDPC code and BCH code.

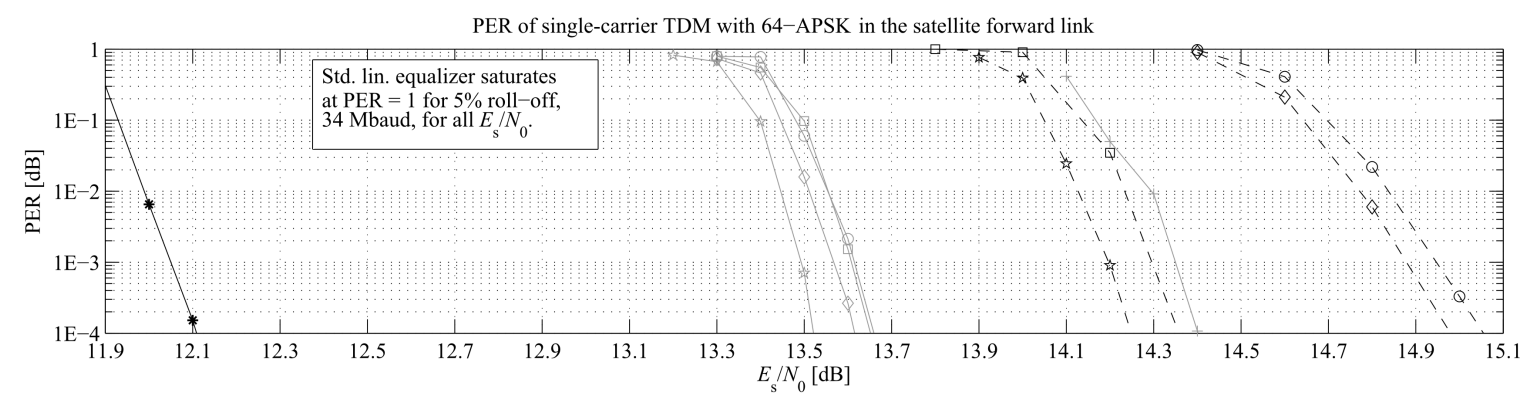

(e) PER of single-carrier TDM with 64-APSK with 3/4-rate LDPC code and BCH code. 


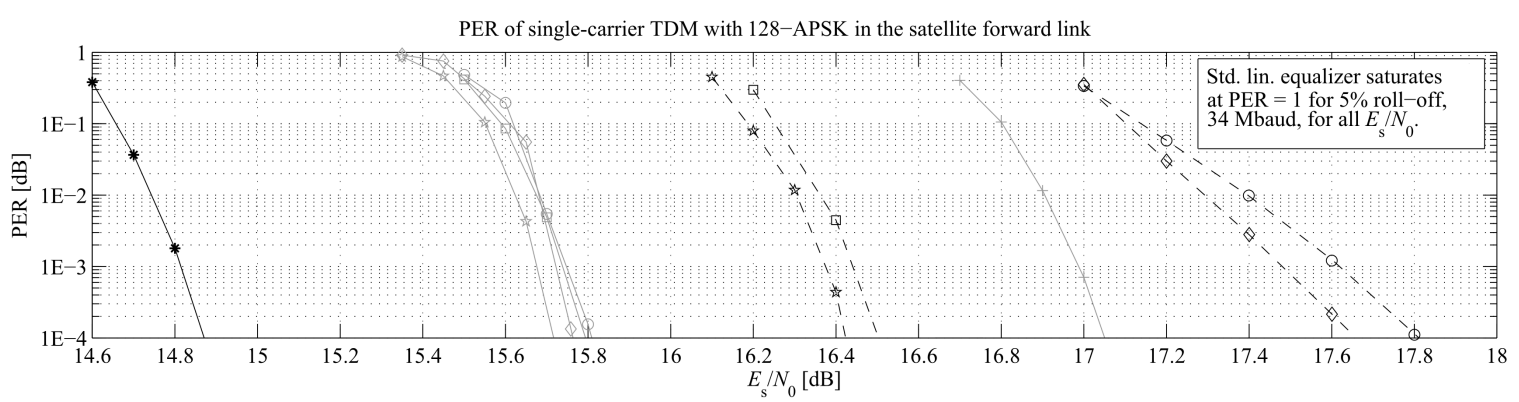

(f) PER of single-carrier TDM with 128-APSK with 3/4-rate LDPC code and BCH code.

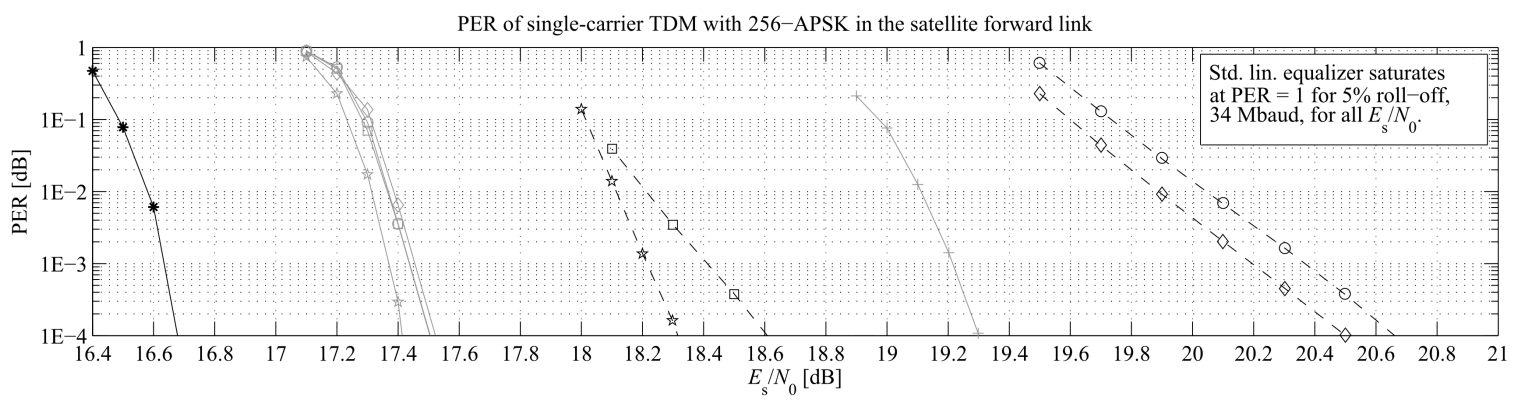

(g) PER of single-carrier TDM with 256-APSK with 3/4-rate LDPC code and BCH code.

Fig. 5: PER of single-carrier TDM in the Ka-band VSAT satellite forward link. The 2 realizations of the proposed equalizer are evaluated for 27.5 MBaud symbol rate with $20 \%$ roll-off and $34 \mathrm{MBaud}$ symbol rate with $5 \%$ roll-off in a $36-\mathrm{MHz}$ transponder.

originally buffered received symbols. The improved vector of received symbols after the $i$ th iteration can be expressed as:

$$
\mathbf{x}^{(i)}=\mathbf{x}^{(0)}-\mathbf{d}^{(i)} .
$$

The output of this process are the newly obtained received symbols after a number of iterations. The complexity is linear with the number of iterations and with the number of processed symbols. It is shown in Section IV that even one iteration can yield a significant energy efficiency gain. Finally, the buffer is released and a new frame of received symbols is processed.

\section{Performance Evaluation}

The performance of the proposed equalizer is evaluated in a Monte Carlo simulation of the PER in the satellite forward link. A single-carrier VSAT scenario is considered as specified in the DVB-S2X implementation guidelines [2]. A symbol rate of 27.5 MBaud is applied with $20 \%$ roll-off as a legacy scenario, while also 34 MBaud with $5 \%$ roll-off is considered as an example of high symbol rate with low roll-off in the transponder bandwidth of $36 \mathrm{MHz}$. Quadrature phase shift keying (QPSK), 8-level phase shift keying (8-PSK), 16-APSK, 32-APSK, 64-APSK, 128-APSK, and 256-APSK constellations are simulated. Corresponding OBO values of $0.8 \mathrm{~dB}, 1 \mathrm{~dB}, 1.8 \mathrm{~dB}, 2.4 \mathrm{~dB}, 3 \mathrm{~dB}, 3.6 \mathrm{~dB}$, and $4.5 \mathrm{~dB}$ are considered, which are lower than the ones used in [2], improving the energy efficiency.

A large number of frames are simulated, e.g., up to $10^{6}$ packets per simulation point, resulting in sufficient convergence of the statistics down to PER of $10^{-4}$. State-of-the-art static data pre-distortion at the transmitter and linear equalization at the receiver are used as benchmarks. In addition, the

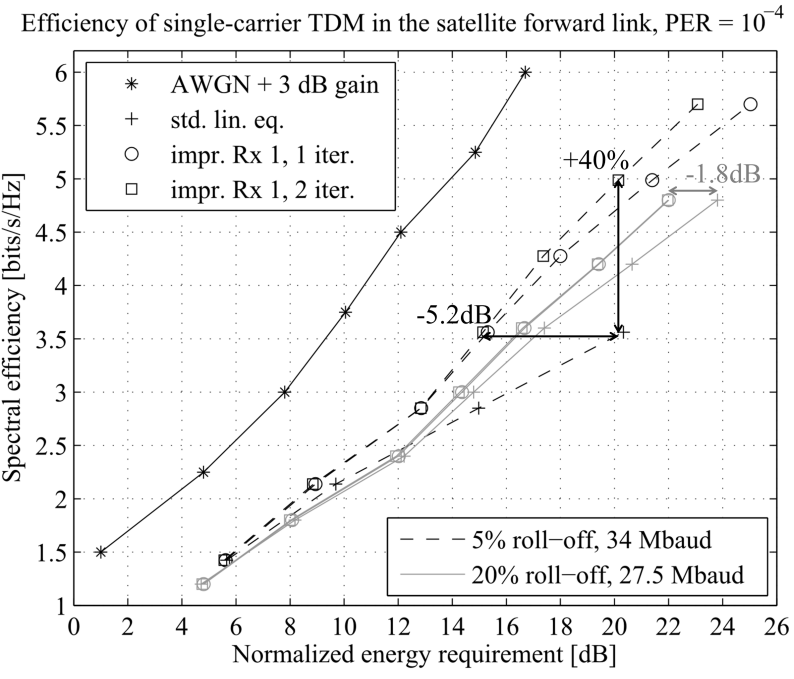

Fig. 6: Spectral efficiency vs. normalized energy requirement of single-carrier TDM with the iterative symbol-based equalizer in the satellite forward link ( $\mathrm{Rx} 1)$.

two realizations of the symbol-based equalizer are evaluated with 1 , and 2 iterations of non-linear noise cancellation. In order to illustrate the SNR penalty of the non-linear satellite channel, the linear channel with AWGN is also simulated as a reference, whereby the gain of $3 \mathrm{~dB}$ in the linear region of the TWTA from Fig. 3 is considered.

The PER results are presented in Fig. 5. The SNR requirement for a PER target is defined as the the ratio of the energy per symbol to noise power spectral density, $E_{\mathrm{S}} / N_{0}$. The reference performance in the linear channel matches the 


\begin{tabular}{|l|l|l|l|l|l|l|l|}
\hline Scenario & QPSK & 8-PSK & 16-APSK & 32-APSK & 64 -APSK & 128-APSK & 256 -APSK \\
\hline 20\% roll-off, 27.5 MBaud & 0.1 & 0.2 & 0.3 & 0.6 & 0.9 & 1.3 & 1.9 \\
\hline $5 \%$ roll-off, 34 MBaud & 0.2 & 0.9 & 2.2 & 5.2 & enabled & enabled & enabled \\
\hline
\end{tabular}

TABLE I: SNR gains $[\mathrm{dB}]$ at $10^{-4}$ PER of 2 iterations of non-linear distortion noise cancellation (Rx 2) relative to standard static pre-distortion and linear equalization in the satellite forward link with a $36-\mathrm{MHz}$ transponder.

results in [11] up to the considered $3-\mathrm{dB}$ gain. In addition, the performance of the scenario with 34-MBaud symbol rate and $5 \%$ roll-off is very close to the results of the DTH scenario in [2]. Except for only the case of QPSK, where only Rx 1 is behind by $0.1 \mathrm{~dB}$ in the case of 27.5-MBaud symbol rate and $20 \%$ roll-off, the proposed equalizer consistently outperforms the standard compensation techniques. The energy efficiency gains of the improved receiver are shown to increase for higher-order modulations due to their higher sensitivity to nonlinear distortion. The SNR gains at PER of $10^{-4}$ of two iterations of non-linear noise cancellation relative to the standard compensation techniques are summarized in TABLE I. The proposed equalizer enables the application of 64-APSK, 128APSK and 256-APSK at $10^{-4}$ PER in the high symbol rate scenario. There is a negligible difference between $\mathrm{Rx} 1$ and Rx 2 within $0.3 \mathrm{~dB}$ across the scenarios. This shows that simple ML demodulation in the detector in the cancellation loop is sufficient, resulting in significant reduction of the computational complexity of the receiver, which is dominated by the soft demapper and the decoder.

The spectral efficiency of the waveforms is plotted against the normalized energy requirement in Fig. 6. In this study, the normalized energy requirement is defined as the summation of SNR requirement and the OBO of the TWTA in log-domain, while the spectral efficiency is defined as $\mathrm{SE}=r \log _{2}(M) R_{\mathrm{s}} / W_{\text {ref }}$, where $r$ is the code rate, $R_{\mathrm{s}}$ is the symbol rate, and $W_{\text {ref }}$ is the transponder bandwidth. Due to its significantly lower complexity and close performance to $\operatorname{Rx} 2$, only the $\operatorname{Rx} 1$ realization of the proposed equalizer is compared to the standard compensation techniques in the nonlinear channel, as well as to the linear channel. It is shown that the improved receiver significantly increases the spectral efficiency of the waveforms, and it is particularly suitable for higher-order modulation. As compared to the standard compensation techniques, an increase of up to $1.8 \mathrm{~dB}$ of the energy efficiency is expected in the scenario with symbol rate of 27.5 MBaud. In the scenario with $34 \mathrm{MBaud}$ symbol rate, a $5.2-\mathrm{dB}$ increase of the energy efficiency are shown at 3.5 bits $/ \mathrm{s} / \mathrm{Hz}$, while an increase of up to $40 \%$ of the spectral efficiency is expected, reducing the gap to the linear channel.

\section{CONCLusion}

In this paper, symbol-based equalization with non-linear noise cancellation has been proposed as an addition to the state-of-the-art linear equalizer at the user terminal receiver in the DVB-S2X satellite forward link. The improved receiver is particularly suitable for application with the recently specified DVB-S2X reference scenarios, including wideband carriers with low roll-off factors down to $5 \%$, high symbol baud rates, and high-order modulations. The performance of the receiver has been assessed for a single-carrier TDM waveform with a comprehensive model of the satellite forward link channel, including the IMUX and OMUX filter responses at the satellite transponder, the non-linear TWTA characteristics, and the phase noise at the user terminal. As compared to stateof-the-art compensation techniques, such as pre-distortion at transmitter and linear equalization at receiver, the improved receiver presented significant gains in the energy efficiency and spectral efficiency. It enables the modulation orders of 64-APSK, 128-APSK, and 256-APSK in a scenario with 34MBaud symbol rate in a $36-\mathrm{MHz}$ transponder with $5 \%$ roll-off, resulting in an increase of both user rates and system capacity. A 5.2-dB increase of the energy efficiency is shown with 32APSK or up to $40 \%$ increase of the spectral efficiency due to the enabled application of 128-APSK. In a scenario with 27.5MBaud symbol rate with $20 \%$ roll-off, up to 1.8 -dB increase of the energy efficiency at 256 -APSK is presented.

\section{REFERENCES}

[1] Second Generation Framing Structure, Channel Coding and Modulation Systems for Broadcasting, Interactive Services, News Gathering and Other Broadband Satellite Applications; Part II: S2-Extensions (DVB$S 2 X)$, Digital Video Broadcasting (DVB) Std. ETSI EN 302 307-2, Oct. 2014.

[2] Implementation Guidelines for the Second Generation System for Broadcasting, Interactive Services, News Gathering and Other Broadband Satellite Applications; Part II: S2-Extensions (DVB-S2X), Digital Video Broadcasting (DVB) Std. ETSI TR 102 376-2, Mar. 2015.

[3] E. Casini, R. De Gaudenzi, and A. Ginesi, "DVB-S2 modem algorithms design and performance over typical satellite channels," Int. J. Satellite Commun. Netw., vol. 22, pp. 281-318, Jun. 2004.

[4] G. Karam and H. Sari, "Analysis of predistortion, equalization, and ISI cancellation techniques in digital radio systems with nonlinear transmit amplifiers," IEEE Trans. Commun., vol. 37, no. 12, pp. 1245-1253, Dec. 1989.

[5] L. Ding, G. T. Zhou, D. R. Morgan, Z. Ma, J. S. Kenney, J. Kim, and C. R. Giardina, "A robust digital baseband predistorter constructed using memory polynomials," IEEE Trans. Commun., vol. 52, no. 1, pp. 159-165, Jan. 2004.

[6] D. Zhou and V. E. DeBrunner, "Novel adaptive nonlinear predistorters based on the direct learning algorithm," IEEE Trans. on Signal Proces., vol. 55, no. 1, pp. 120-133, Jan. 2007.

[7] N. Kelly, M. Allegue-Martinez, P.-D. Arapoglou, and A. Zhu, "Bandwidth-constrained digital pre-distortion technique for multi-carrier satellite communications," Int. J. Satellite Commun. Netw., Apr. 2015.

[8] J. Proakis, Digital Communications, 4th ed. McGraw Hill, Aug. 2000.

[9] D. Ampeliotis, A. A. Rontogiannis, K. Berberidis, M. Papaleo, and G. E. Corazza, "Turbo equalization of non-linear satellite channels using soft interference cancellation," in Proc. 4th Advanced Satellite Mobile Systems Conference (ASMS2008), Bologna, Italy, Aug. 26-28 2008, pp. 289-292.

[10] S. Dimitrov, "Iterative cancellation of non-linear distortion noise in digital communication systems," IEEE Trans. Commun., vol. 63, no. 6, pp. 2325-2336, Jun. 2015.

[11] B. Azarbad and A. B. Sali, DVB-S2 Model in Matlab: Issues and Impairments, MATLAB - A Fundamental Tool for Scientific Computing and Engineering Applications, V. Katsikis, Ed. InTech, 2012, vol. 2. 